\title{
FLOATING BODIES AND ILLUMINATION BODIES
}

\author{
ELISABETH WERNER * \\ Department of Mathematics \\ Case Western Reserve University \\ Cleveland, Ohio 44106, U. S. A. \\ and \\ Université de Lille 1 \\ UFR de Mathématique \\ 59655 Villeneuve d'Ascq, France \\ E-mail: emw2@po.cwru.edu
}

\begin{abstract}
We give an overview over certain geometric bodies, the floating body and the illumination body, associated to a convex body. We also introduce an analytic property for a convex bodies, the affine surface area. We describe connections between the introduced concepts.
\end{abstract}

\section{Introduction and Notations}

We give an overview over certain geometric bodies associated to a convex body and a certain analytic property defined for a convex body. We describe connections between the introduced concepts. We omit most of the proofs as they have been given in the original papers and we refer to the original articles for the proofs.

We use the following notations. $m$ is the Lebesgue measure on $\mathbb{R}^{n}$. $B_{2}^{n}(a, r)$ is the n-dimensional Euclidean ball with radius $r$ centered at $a$. We put $B_{2}^{n}=B_{2}^{n}(0,1)$. By $\|$. $\|$ we denote the standard Euclidean norm on $\mathbb{R}^{n}$, by $\left\langle\cdot,>\right.$ the standard inner product on $\mathbb{R}^{n}$. For two points $x$ and $y$ in $\mathbb{R}^{n}[x, y]=\{\alpha x+(1-\alpha) y: 0 \leq \alpha \leq 1\}$ denotes the line segment from $x$ to $y$.

For a convex body $K$ in $\mathbb{R}^{n}, \stackrel{\circ}{K}$ is the interior of $K$ and $\partial K$ is the boundary

* partially supported by a nsf grant, by a nato collaborative linkage grant and by a nsf advance opportunity grant. 
of $K$. We also write $S^{n-1}$ for $\partial B_{2}^{n}$. For $x \in \partial K, N(x)$ is the outer unit normal vector to $\partial K$ in $x$. It may not be unique.

$H(x, \xi)$ is the hyperplane containing the point $x$ and orthogonal to $\xi$. $H^{-}(x, \xi)$ is the closed halfspace containing the point $x+\xi, H^{+}(x, \xi)$ the other halfspace.

Now we introduce the geometric bodies associated to a given convex body. Let $K$ be a convex body in $\mathbb{R}^{n}$ and let $t \in \mathbb{R}, t \geq 0$.

The floating body $K_{t}$ of $K$ is the intersection of all halfspaces $H^{+}$whose defining hyperplanes $H$ cut off a set of volume $t$ from $K$

$$
K_{t}=\bigcap_{\int_{K \cap H^{-}} d m \leq t} H^{+}
$$

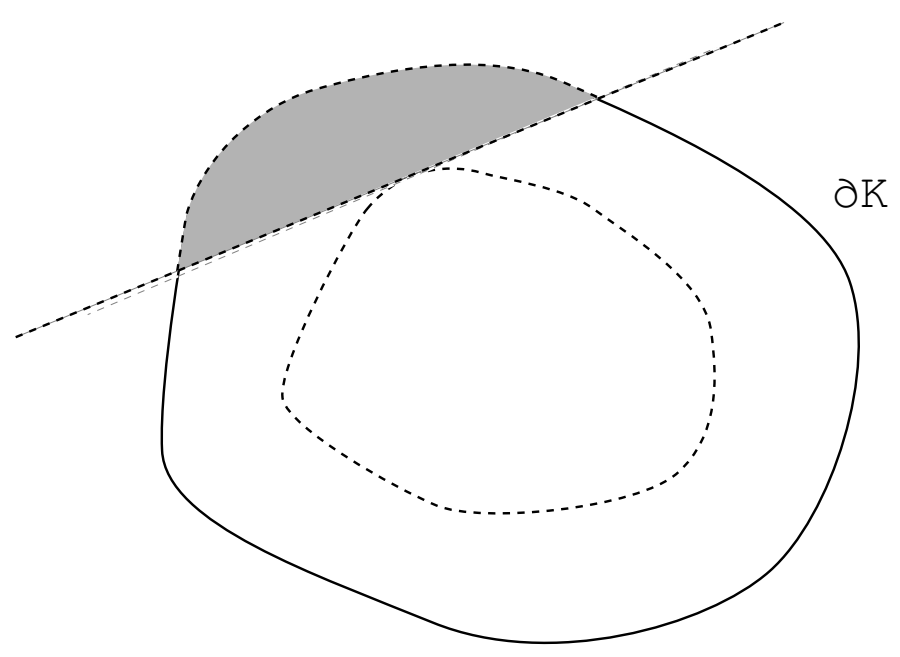

The hyperplane cuts off a set of volume $\dagger$

Figure 1

We choose $t$ small enough so that $K_{t} \neq \emptyset$.

The illumination body $K^{t}$ of $K$ 


$$
K^{t}=\left\{x \in \mathbb{R}^{n}: \operatorname{vol}_{n}(c o[x, K]) \leq t\right\}
$$

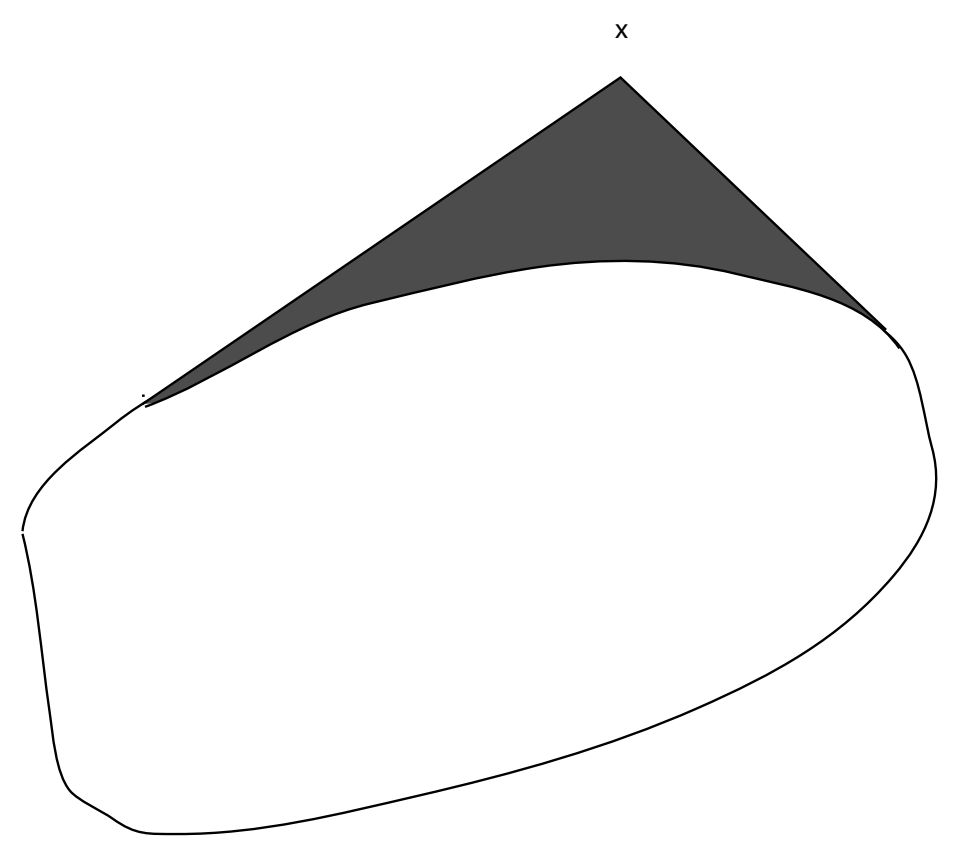

Figure 2:

$\operatorname{vol}_{n}(c o[x, K]) \leq t$

The concept of floating body goes as far back as Dupin and Blaschke ${ }^{3}$. They considered only dimenensions 2 and 3. Leichtweiss ${ }^{9}$ extended Blaschke's definition to $\mathbb{R}^{n}$. This definition defines the "boundary" of the floating body $K_{[t]}$ as the set of points that are the center of gravity of all hyperplanes $H$ such that $K \cap H^{-}=t$. This floating body $K_{[t]}$ is different from the one introduced above. It need not be convex as one can see by considering a triangle in $\mathbb{R}^{2}$ (see ${ }^{10}$ ). It is clear that the above floating body $K_{t}$ is convex as the intersection of convex sets. As far as we know, the the floating body $K_{t}$ was introduced by Schütt and Werner in ${ }^{19}$.

It is obvious that $K_{0}=K$ and that $K^{0}=K$ and that for all $t \geq 0$ $K_{t} \subseteq K$ and $K \subseteq K^{t}$. Further properties are 


\section{Proposition 1.}

Let $K$ be a convex body in $\mathbb{R}^{n}$. Then we have

(i) For t small enough, $B_{2}^{n}(a, r)_{t}$ is again a Euclidean ball centered at a with radius

$$
r\left(1-\frac{1}{2}\left(\frac{(n+1) t}{f_{1}\left(\frac{\Delta}{r}\right) r^{n} \operatorname{vol}_{n-1}\left(B_{2}^{n-1}\right)}\right)^{\frac{2}{n+1}}\right),
$$

where $f_{1}: \mathbb{R}^{+} \rightarrow \mathbb{R}^{+}$is a continuous function such that $\lim _{s \rightarrow 0} f_{1}(s)=1$.

(ii) Through every point of $\partial K_{t}$ there is at least one hyperplane that cuts off a set of volume $t$ from $K$.

(iii) A supporting hyperplane $H$ of $K_{t}$ that cuts off a set of volume $t$ touches $K_{t}$ in exactly one point, namely the barycenter of $K \cap H$.

(iv) $K_{t}$ is strictly convex.

(v) Let

$$
t_{0}=\inf \left\{t: \operatorname{vol}_{n}\left(K_{t}\right)>0\right\}
$$

Then $K_{t_{0}}$ consists of one point only.

(vi) $K_{t}$ is invariant under affine transformations with determinant 1.

\section{Proof.}

(i) By symmetry $B_{2}^{n}(a, r)_{t}$ is again a Euclidean ball centered at $a$. For $t$ small, we now compute the radius $\rho_{1}$ of this ball. Let $H$ be a hyperplane that cuts off $t$ of $B_{2}^{n}(a, r)$ and let $\Delta$ be the height of the cap $B_{2}^{n}(a, r) \cap H^{-}$. Then

$$
t=r^{n} \operatorname{vol}_{n-1}\left(B_{2}^{n}\right) \int_{1-\frac{\Delta}{r}}^{1}\left(1-x^{2}\right)^{\frac{n-1}{2}} d x=\frac{2^{\frac{n+1}{2}}}{n+1} f_{1}\left(\frac{\Delta}{r}\right) r^{\frac{n-1}{2}} \Delta^{\frac{n+1}{2}},
$$

where $f_{1}: \mathbb{R}^{+} \rightarrow \mathbb{R}^{+}$is a continuous function such that $\lim _{s \rightarrow 0} f_{1}(s)=1$. Hence

$$
\rho_{1}=r-\Delta=r\left(1-\frac{1}{2}\left(\frac{(n+1) t}{f_{1}\left(\frac{\Delta}{r}\right) r^{n} \operatorname{vol}_{n-1}\left(B_{2}^{n-1}\right)}\right)^{\frac{2}{n+1}}\right) .
$$

(ii) Let $x \in \partial K_{t}$. We choose a sequence $x_{k}, k \in \mathbb{N}$, such that $x_{k} \notin$ $K_{t}$ and $\lim _{k \rightarrow \infty} x_{k}=x$. By the definition of $K_{t}$ we find for every $x_{k}$ a 
hyperplane $H_{k}$ such that $x_{k} \in H_{k}^{-}$and $\operatorname{vol}_{n}\left(K \cap H_{k}^{-}\right)=t$. By compactness there is a subsequence $H_{k_{j}}, j \in \mathbb{N}$, that converges to a hyperplane $H$. Therefore $\operatorname{vol}_{n}\left(K \cap H^{-}\right)=t$ and $x \in H$.

(iii) Let $y \in \partial K_{t}$ and let $H=H(y, \xi)$ be a supporting hyperplane of $K_{t}$ that cuts off $t$ from $K$. The barycenter of $K \cap H$ is computed by

$$
z=\frac{1}{\operatorname{vol}_{n-1}(K \cap H)} \int_{K \cap H} x d x
$$

Suppose $z \neq y$. We choose

$$
\eta=\frac{\xi+\epsilon(y-z)}{\|\xi+\epsilon(y-z)\|}
$$

We have

$$
\begin{gathered}
\operatorname{vol}_{n}\left(K \cap H^{-}(y, \eta)\right)= \\
\operatorname{vol}_{n}\left(K \cap H^{-}(y, \xi)\right)+\operatorname{vol}_{n}\left(K \cap H^{+}(y, \xi) \cap H^{-}(y, \eta)\right)-\operatorname{vol}_{n}\left(K \cap H^{+}(y, \eta) \cap H^{-}(y, \xi)\right)
\end{gathered}
$$

We estimate now the volume of the two wedges. Let

$$
h(x)=\sup \left\{s: x-s \xi \in K \cap H^{+}(y, \xi) \cap H^{-}(y, \eta)\right\}
$$

Let $\alpha=\alpha(\epsilon)$ be the angle between the two planes. For small $\alpha$ we get that

$$
\operatorname{vol}_{n}\left(K \cap H^{+}(y, \xi) \cap H^{-}(y, \eta)\right)=\int_{K \cap H(y, \xi) \cap H^{-}(y, \eta)} h(x) d x+O\left(\alpha^{2}\right) .
$$

which, for small $\alpha$, equals

$$
\tan \alpha \int_{K \cap H(y, \xi) \cap H^{-}(y, \eta)}\left|\left\langle\frac{y-z}{\|y-z\|}, x-y\right\rangle\right| d x+O\left(\alpha^{2}\right)
$$

The other wedge is handled in the same way and we get that the volume

$$
\operatorname{vol}_{n}\left(K \cap H^{-}(y, \eta)\right)
$$

for small $\alpha$ equals

$$
\begin{gathered}
\operatorname{vol}_{n}\left(K \cap H^{-}(y, \xi)\right)+\tan \alpha \int_{K \cap H(y, \xi) \cap H^{-}(y, \eta)}\left|\left\langle\frac{y-z}{\|y-z\|}, x-y\right\rangle\right| d x \\
-\tan \alpha \int_{K \cap H(y, \xi) \cap H^{+}(y, \eta)}\left|\left\langle\frac{y-z}{\|y-z\|}, x-y\right\rangle\right| d x+O\left(\alpha^{2}\right)
\end{gathered}
$$

This equals

$$
\operatorname{vol}_{n}\left(K \cap H^{-}(y, \xi)\right)+\tan \alpha\left\langle\frac{y-z}{\|y-z\|}, \int_{K \cap H(y, \xi)}(x-y) d x\right\rangle+O\left(\alpha^{2}\right)=
$$




$$
\begin{gathered}
\operatorname{vol}_{n}\left(K \cap H^{-}(y, \xi)\right)+\tan \alpha \operatorname{vol}_{n-1}(K \cap H(y, \xi))\left\langle\frac{y-z}{\|y-z\|}, z-y\right\rangle+O\left(\alpha^{2}\right)= \\
\operatorname{vol}_{n}\left(K \cap H^{-}(y, \xi)\right)-\tan \alpha \operatorname{vol}_{n-1}(K \cap H(y, \xi))\|z-y\|+O\left(\alpha^{2}\right)
\end{gathered}
$$

This means that for sufficiently small $\alpha$ this expression is strictly smaller than

$$
\operatorname{vol}_{n}\left(K \cap H^{-}(y, \xi)\right)
$$

Therefore the hyperplane $H(y, \eta)$ cuts off a set of volume strictly smaller than $t$. We choose a hyperplane that is parallel to $H(y, \eta)$ and cuts off a set of volume $t$. But then $y$ is also cut off and cannot be an element of $K_{t}$.

(iv) follows from (iii).

\section{Proposition 2.}

Let $K$ be a convex body in $\mathbb{R}^{n}$. Then we have

(i) $K^{t}$ is convex.

(ii) For $t$ small enough, $B_{2}^{n}(a, r)^{t}$ is again a Euclidean ball centered at $a$ with radius

$$
\frac{r}{1-\left(\frac{n(n+1) t}{f_{2}\left(\frac{r}{\rho_{2}}\right) r^{n} \operatorname{vol}_{n-1}\left(B_{2}^{n-1}\right)}\right)^{\frac{2}{n+1}}}
$$

where $f_{2}: \mathbb{R}^{+} \rightarrow \mathbb{R}^{+}$is a continuous function such that $\lim _{s \rightarrow 1} f_{2}(s)=$ $2^{\frac{n+1}{2}}$.

(iii) $K^{t}$ is invariant under affine transformations with determinant 1 .

(iv) For a polytope $P$ in $\mathbb{R}^{n}, P^{t}$ is again a polytope.

\section{Proof.}

(i) To show that the illumination body is convex, it is enough to show that the illumination body of a polytope is convex as every convex body in 
$\mathbb{R}^{n}$ can be approximated by polytopes. Let $P^{t}$ be the illumination body of a polytope $P$. Let $x, y \in \partial P^{t}$. Then

$$
\begin{aligned}
t & =\frac{1}{n} \sum_{\{F: F \text { face of } P\}} \operatorname{vol}_{n-1}(F) \max \left\{\left\langle N_{F}, x-c_{F}>, 0\right\}\right. \\
& =\frac{1}{n} \sum_{\{F: F \text { face of } P\}} \operatorname{vol}_{n-1}(F) \max \left\{<N_{F}, y-c_{F}>, 0\right\},
\end{aligned}
$$

where $N_{F}$ is the outer normal and $c_{F}$ is the center of gravity of the face $F$. Let $0<\lambda<1$ and let $z=\lambda x+(1-\lambda) y$. Then

$$
\begin{aligned}
& \operatorname{vol}_{n}([z, P]) \leq \frac{1}{n} \sum_{\{F: F \text { face of } P\}} \operatorname{vol}_{n-1}(F) \max \left\{<N_{F}, z-c_{F}>, 0\right\} \\
& \quad \leq \frac{\lambda}{n} \sum_{\{F: F \text { face of } P\}} \operatorname{vol}_{n-1}(F) \max \left\{<N_{F}, x-c_{F}>, 0\right\}+ \\
& \frac{1-\lambda}{n} \sum_{\{F: F \text { face of } P\}} \operatorname{vol}_{n-1}(F) \max \left\{<N_{F}, y-c_{F}>, 0\right\}=t .
\end{aligned}
$$

(ii) Again, by symmetry, $B_{2}^{n}(a, r)^{t}$ is a Euclidean ball centered at $a$. For $t$ small, we now compute the radius $\rho_{2}$ of this ball. $t$ equals the volume of a cone minus the volume of a cap. Hence with Proposition 1 (i) we get that

$$
\begin{gathered}
t=\operatorname{vol}_{n-1}\left(B_{2}^{n-1}\right)\left(\frac{r^{n-1} \rho_{2}}{n}\left(1-\frac{r^{2}}{\rho_{2}^{2}}\right)^{\frac{n+1}{2}}-\frac{2^{\frac{n+1}{2}} r^{n}}{n+1} f_{1}\left(1-\frac{r}{\rho_{2}}\right)\left(1-\frac{r}{\rho_{2}}\right)^{\frac{n+1}{2}}\right) \\
=\frac{r^{n}}{n(n+1)} f_{2}\left(\frac{r}{\rho_{2}}\right)\left(1-\frac{r}{\rho_{2}}\right)^{\frac{n+1}{2}} \operatorname{vol}_{n-1}\left(B_{2}^{n-1}\right),
\end{gathered}
$$

where $f_{2}: \mathbb{R}^{+} \rightarrow \mathbb{R}^{+}$is a continuous function such that $\lim _{s \rightarrow 1} f_{2}(s)=2^{\frac{n+1}{2}}$. Hence

$$
\rho_{2}=\frac{r}{1-\left(\frac{n(n+1) t}{f_{2}\left(\frac{r}{\rho_{2}}\right) r^{n} \operatorname{vol}_{n-1}\left(B_{2}^{n-1}\right)}\right)^{\frac{2}{n+1}}} .
$$


Floating bodies and illumination bodies are -in a sense- dual notions. But notice that in general it is not the case that $\left(K_{t}\right)^{0}=\left(K^{0}\right)^{t}$ Indeed, for a polytope $P, P^{0}$ is a polytope and hence by Proposition 2 (iv), $\left(P^{0}\right)^{t}$ is a polytope. However for a polytope $P, P_{t}$ is strictly convex by Proposition 1 (iv), hence not a polytope.

\section{Affine surface area}

The affine surface area was originally introduced by Blaschke ${ }^{3}$ for convex bodies in $\mathbb{R}^{3}$ with sufficiently smooth boundary. Its definition involves the Gauss curvature of the boundary points of a convex body. Hence it provides a tool to "measure" the boundary structure of a convex body. Therefore it is not surprising that the affine surface area occurs naturally in problems related to the boundary of a convex body, so for instance in the approximation of convex bodies by polytops. For more information about this subject and the role the affine surface area plays there, we refer to the works by Bárány, ${ }^{1,2}$, Gruber ${ }^{5,6,7}$, Schütt ${ }^{17,18}$ and Schütt and Werner ${ }^{20}$.

Extensions of the affine surface area to higher dimensions and arbitrary convex bodies were only found much later than Blaschke's times by Leichtweiss ${ }^{9}$, Lutwak ${ }^{11,12}$, Schütt and Werner ${ }^{19}$, Schmuckenschläger ${ }^{16}$, Meyer and Werner ${ }^{15}$ and Werner ${ }^{24}$. Additional references to the affine surface area as well as the proofs of the facts mentioned without their proofs and further applications can also be found in those papers as well as in $14,8,25$.

Let $K$ be a convex body in $\mathbb{R}^{n}$. The affine surface area $a s(K)$ is

$$
a s(K)=\int_{\partial K} \kappa(x)^{\frac{1}{n+1}} d \mu(x),
$$

where $\mu$ is the surface measure on $\partial K, \kappa$ the (generalized) Gaussian curvature.

\section{Examples}

(i) For every convex polytope $P$ in $\mathbb{R}^{n}$, as $(P)=0$. This holds as a.e. on $\partial P$ the Gauss curvature is equal to 0.

(ii) Let $1 \leq p<\infty$ and let $B_{p}^{n}=\left\{x \in \mathbb{R}^{n}:\|x\|_{p}=\left(\sum_{i=1}^{n}\left|x_{i}\right|^{p}\right)^{\frac{1}{p}} \leq 1\right\}$ be the $l_{p}^{n}$-unit ball. Then, for $1<p<\infty$, we have for the affine surface area of $B_{p}^{n}$ 


$$
\operatorname{as}\left(B_{p}^{n}\right)=\frac{2^{n}(p-1)^{\frac{n-1}{n+1}}\left(\Gamma\left(\frac{p-2}{p(n+1)}+\frac{1}{p}\right)\right)^{n-1} \Gamma\left(\frac{p+n-1}{(n+1) p}\right)}{p^{n-1} \Gamma\left(\frac{p+n-1}{(n+1) p}+(n-1) \frac{1}{p}\left(\frac{p-2}{n+1}+1\right)\right)}
$$

For $p=2$ we get

$$
a s\left(B_{2}^{n}\right)=2 \frac{\Gamma\left(\frac{1}{2}\right)^{n}}{\Gamma\left(\frac{n}{2}\right)}=n \frac{\pi^{\frac{n}{2}}}{\Gamma\left(1+\frac{n}{2}\right)}=\operatorname{vol}_{n-1}\left(\partial B_{2}^{n}\right)
$$

and for $p=1$ and for $p=\infty$ we get that

$$
\operatorname{as}\left(B_{1}^{n}\right)=0 \text { and } \operatorname{as}\left(B_{\infty}^{n}\right)=0,
$$

as $B_{1}^{n}$ and $B_{\infty}^{n}=\left\{x \in \mathbb{R}^{n}:\|x\|_{\infty}=\max _{1 \leq i \leq n}\left|x_{i}\right| \leq 1\right\}$ are polytopes.

\section{Proof} is

The Gauss-Kronecker curvature at $x \in \partial B_{p}^{n}$ with positive coordinates

$$
\kappa(x)=\frac{(p-1)^{n-1}\left(\prod_{i=1}^{n} x_{i}^{p-2}\right)}{\left(\sum_{i=1}^{n} x_{i}^{2 p-2}\right)^{\frac{n+1}{2}}}
$$

Thus we get

$$
\begin{gathered}
a s\left(B_{p}^{n}\right)=(p-1)^{\frac{n-1}{n+1}} \int_{\partial B_{p}^{n}} \frac{\left(\prod_{i=1}^{n}\left|x_{i}\right|^{p-2}\right)^{\frac{1}{n+1}}}{\left(\sum_{i=1}^{n}\left|x_{i}\right|^{2 p-2}\right)^{\frac{1}{2}}} d \mu(x)= \\
2^{n}(p-1)^{\frac{n-1}{n+1}} \int_{B_{p}^{n-1+}}\left(\prod_{i=1}^{n-1} x_{i}^{p-2}\right)^{\frac{1}{n+1}}\left(1-\sum_{i=1}^{n-1} x_{i}^{p}\right)^{\frac{n-n p-1}{(n+1) p}} d\left(x_{1}, \ldots, x_{n-1}\right)
\end{gathered}
$$

By ${ }^{4}$ this equals

$$
\begin{gathered}
2^{n}(p-1)^{\frac{n-1}{n+1}} p^{-n+1} \frac{\left(\Gamma\left(\frac{p-2}{p(n+1)}+\frac{1}{p}\right)\right)^{n-1}}{\Gamma\left(1-\frac{n p+1-n}{(n+1) p}+(n-1) \frac{1}{p}\left(\frac{p-2}{n+1}+1\right)\right)} \Gamma\left(1-\frac{n p+1-n}{(n+1) p}\right)= \\
\frac{2^{n}(p-1)^{\frac{n-1}{n+1}}\left(\Gamma\left(\frac{p-2}{p(n+1)}+\frac{1}{p}\right)\right)^{n-1} \Gamma\left(\frac{p+n-1}{(n+1) p}\right)}{p^{n-1} \Gamma\left(\frac{p+n-1}{(n+1) p}+(n-1) \frac{1}{p}\left(\frac{p-2}{n+1}+1\right)\right)}
\end{gathered}
$$

The affine surface area has many nice properties which make it useful for applications. In Proposition 3 we mention some of them. A map $T$ from 
the set of all convex bodies in $\mathbb{R}^{n}$ into $\mathbb{R}$ is called a valuation if we have for all convex bodies $C, K$ such that $C \cup K$ is again convex

$$
T(C \cup K)+T(C \cap K)=T(C)+T(K)
$$

Proposition 3. Let $K$ be a convex body in $\mathbb{R}^{n}$.

(i) affine invariance

$$
\operatorname{as}(T(K))=T(\operatorname{as}(K))
$$

for an affine $T$ with $\operatorname{det} T=1$.

(ii) valuation

If $C, K$, and $C \cup K$ are convex bodies then we have

$$
a s(C \cup K)+a s(C \cap K)=a s(C)+a s(K)
$$

(iii) affine isoperimetric inequality

$$
\operatorname{as}(K) \leq n \operatorname{vol}_{n}(K)^{\frac{n-1}{n+1}} \operatorname{vol}_{n}\left(B_{2}^{n}\right)^{\frac{2}{n+1}},
$$

with equality iff $K$ is an ellipsoid.

As the affine surface area is related to the boundary structure of of a convex body it comes in naturally in questions of approximation of convex bodies by polytopes:

as $(\mathrm{K})$ "measures" how to distribute the vertices of the approximating polytope on $\partial K$ for optimal approximation (see f.i. ${ }^{1,2,5,6,7,20}$ ).

Other applications are to characterizations of ellipsoids ${ }^{21,22}$, differential equations ${ }^{13}$, geometric flows ${ }^{22}$, computer vision ${ }^{23}$.

Next we want to indicate how the concepts of floating body respectively illumination body and affine surface area are related.

\section{Theorem 4. 19}

Let $K$ be a convex body in $\mathbb{R}^{n}$. Then

$$
\lim _{t \rightarrow 0} \frac{\operatorname{vol}_{n}(K)-\operatorname{vol}_{n}\left(K_{t}\right)}{t^{\frac{2}{n+1}}}=c_{n} \int_{\partial K} \kappa(x)^{\frac{1}{n+1}} d \mu(x),
$$

where $c_{n}=\frac{1}{2}\left(\frac{n+1}{\operatorname{vol}_{n-1}\left(B_{2}^{n-1}\right)}\right)^{\frac{2}{n+1}}$ is a constant depending only on $n$. 
and

Theorem 5. ${ }^{24}$

Let $K$ be a convex body in $\mathbb{R}^{n}$. Then

$$
\begin{gathered}
\qquad \lim _{t \rightarrow 0} \frac{\operatorname{vol}_{n}\left(K^{t}\right)-\operatorname{vol}_{n}(K)}{t^{\frac{2}{n+1}}}=d_{n} \int_{\partial K} \kappa(x)^{\frac{1}{n+1}} d \mu(x) . \\
\text { where } d_{n}=\frac{1}{2}\left(\frac{n(n+1)}{\operatorname{vol}_{n-1}\left(B_{2}^{n-1}\right)}\right)^{\frac{2}{n+1}} \text { is a constant depending only on } n .
\end{gathered}
$$

Notice that the above two theorems provide a geometric interpretation of the affine surface area in terms of volume differences of a convex body and its floating body respectively of its illumination body. Thus one can use the left hand sides of Theorems 4 and 5 to define the affine surface area for general convex bodies in $\mathbb{R}^{n}$ and not only for convex bodies with sufficiently smooth boundary as it had been done originally.

\section{References}

1. I. Bárány, Random polytopes in smooth convex bodies, Mathematika 39 (1992), 81-92.

2. I. Bárány, Affine perimeter and limit shape, Journal für Reine und Angew. Math.(1997), 71-84.

3. W. Blaschke, Vorlesungen über Differentialgeometrie II: Affine Differentialgeometrie, Springer Verlag, 1923.

4. I.S. Gradsteyn, I.M. Ryzhik, Table of Integrals, Series, and Products, Academic Press, New York, 1965.

5. P.M. Gruber, Approximation of convex bodies, Convexity and its Applications, Birkhäuser, Basel (1983), 131-162.

6. P. Gruber, Aspects of approximation of convex bodies, Handbook of Convex Geometry vol.A, North Holland (1993), 321-345.

7. P. M. Gruber, Asymptotic estimates for best and stepwise approximation of convex bodies II, Forum Math. 5 (1993), 521-538.

8. D. Hug, Contributions to affine surface area, Manuscripta Math. 91 (1996) no. 3, 283-301.

9. K. Leichtweiss, Über ein Formel Blaschkes zur Affinoberfläche, Studia Scient. Math. Hung. 21 (1986), 453-474.

10. K.Leichtweiss, Affine geometry of convex bodies, Johann Ambrosius Barth Verlag, Heidelberg (1998).

11. E. Lutwak, Extended affine surface area, Adv. in Math. 85 (1991), 39-68.

12. E. Lutwak, The Brunn-Minkowski-Firey Theory II: Affine and Geominimal Surface Areas, Adv. in Math. 118 (1996), 244-294. 
13. E. Lutwak and V. Oliker, On the regularity of solutions to a generalization of the Minkowski problem, J. Differential Geometry 41 (1995), 227-246.

14. M. Ludwig and M. Reitzner, A characterization of affine surface area, Adv. Math. 147 (1999), no. 1, 138-172.

15. M. Meyer and E. Werner, The Santaló-regions of a convex body, Transactions of the AMS 350, no.11 (1998), 4569-4591.

16. M. Schmuckenschläger, The distribution function of the convolution square of a convex symmetric body in $\mathbf{R}^{n}$, Israel J. Math. 78 (1992), 309-334.

17. C. Schütt, Random polytopes and affine surface area, Math. Nachrichten 170 (1994), 227-249.

18. C. Schütt, Floating body, Illumination body, and polytopal approximation, Convex geometric analysis (Berkeley, CA, 1996), 203-229, Math. Sci. Res. Inst. Publ., 34, Cambridge Univ. Press, Cambridge (1999).

19. C. Schütt and E. Werner, The convex floating body, Math. Scand. 66 (1990), 275-290.

20. C. Schütt and E. Werner, Polytopes with vertices chosen randomly from the boundary of a convex body, GAFA 2001-2002, Lecture Notes in Mathematics 1807, Springer Verlag, p. 241-422.

21. C. Schütt and E. Werner, Homothetic floating bodies, Geom. Dedic., 49 (1992), 335-348.

22. A. Stancu, The floating body problem, preprint.

23. A. Tannenbaum, Three Snippets of Curve Evolution Theory in Computer Vision, Math.and Computer Modelling Journal 24 (1996), 103-119.

24. E. Werner, Illumination bodies and affine surface area, Studia Math.110 (1994), 257-269.

25. E. Werner, A general geometric construction of affine surface area, Studia Math.132 (3) (1999), 227-238. 